\title{
Impact of the Floating Body Effect on Noise in SOI Devices Investigated by Hydrodynamic Simulation
}

\author{
C. Jungemann ${ }^{\dagger}$, B. Neinhüs, C. D. Nguyen, and B. Meinerzhagen \\ NST, TU Braunschweig, Postfach 33 29, 38023 Braunschweig, Germany \\ †Phone: +495313913168, Fax: +49531391 8189, \\ Jungemann@ieee.org
}

\begin{abstract}
Without a tie, the body of an SOI devices floats and majority carrier generation causes a kink in the output characteristics. The underlying feedback mechanism strongly amplifies the noise at low frequencies. This effect is investigated for the first time by $2 \mathrm{D}$ bipolar hydrodynamic device simulation including a quantum correction and consistent mobility and noise models for the channel. The simulations reproduce the noise behavior observed experimentally and support the conclusions drawn based on analytical modeling. Not only noise due to impact ionization but also due to other generation/recombination processes is found to be amplified by the floating body effect.
\end{abstract}

\section{Introduction}

The kink effect is a well-known phenomenon in SOI devices without a body tie, but it can occur in any kind of MOS devices (e.g. double gate or tri-gate MOSFETs) if the body is floating. The floating body effect leads to a kink in the output characteristics, because holes generated by impact ionization (II) in an n-type MOS device accumulate in the body and increase the forward bias of the source/body diode leading to feedback. This causes not only the kink in the output characteristics but also a strong increase in low frequency noise [1]. Furthermore, II is not the only source of the kink effect. Other generation phenomena can lead to similar effects. For example, the so-called linear kink effect is due to holes generated by gate tunneling currents [2].

The impact of the floating body effect on noise is investigated in this work for the first time by hydrodynamic device simulation.

\section{Simulation Model and Device Structure}

Recently, the first 2D bipolar hydrodynamic noise model has been developed [3]. It includes a quantum correction and consistent mobility and noise models for the inversion layer and has been shown to yield accurate results for NMOSFETs [4]. The simulations include noise due to carrier scattering (so-called diffusion or thermal noise), due to II (simulated with the local temperature model [5]), and due to Shockley-Read-Hall (SRH) recombination [6, 7]. Because of the lack of a microscopic model for 1/f noise, this type of noise is not considered in the simulations. The simulated SOI NMOSFET has a body thickness of $180 \mathrm{~nm}$, a top oxide of $8.5 \mathrm{~nm}$, a back oxide of $400 \mathrm{~nm}$, and a gate length of 500nm similar to the device in Ref. [1]. The device is of the partially depleted type and has a homogeneous body doping of $2 \cdot 10^{17} / \mathrm{cm}^{-3}$. The output characteristics 


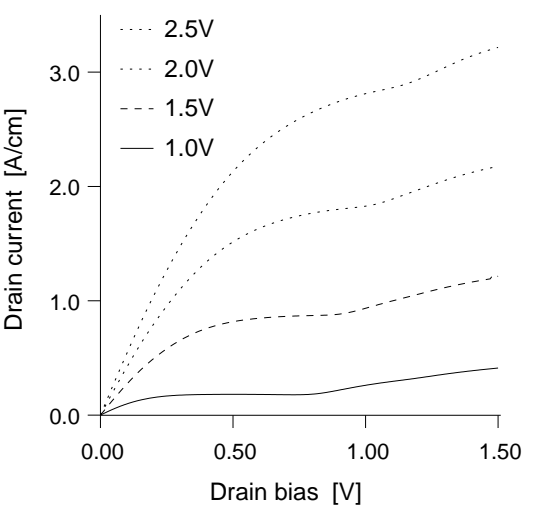

Fig. 1 Output characteristics of the SOI NMOSFET for different gate voltages.

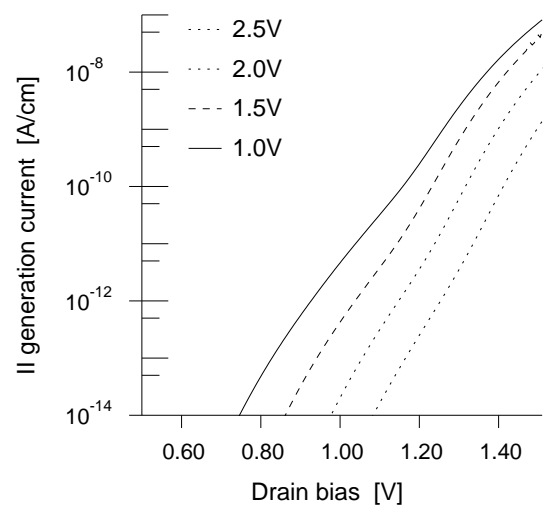

Fig. 2 II generation current of the SOI NMOSFET for different gate voltages.

of the device are shown in Fig. 1 and the kink effect is clearly visible. In Fig. 2 the corresponding total hole current generated by II is shown and the kink occurs, when the generation current is larger than about $10 \mathrm{fA} / \mathrm{cm}$. Even accounting for the built-in voltages, the kink occurs at a drain bias which is about $0.2 \mathrm{~V}$ too low. This might be due to the local temperature model which does not include the threshold effect of II. On the other hand, this small shift in drain bias does not impair the conclusions drawn below and more accurate nonlocal models destroy the band structure of the Jacobian required for noise calculations.

\section{Noise Results}

The floating body effect increases the low frequency noise which is shown in Fig. 3. The additional excess noise is well described by a lorentzian of which the maximum decreases with drain bias and the corner frequency increases similar to experiments [1]. In Figs. 4 and 5 the contributions of the different noise sources to the drain current noise are shown. At a drain bias of $0.6 \mathrm{~V}$, well below the kink, the low frequency noise is dominated by SRH recombination, whereas at $1.2 \mathrm{~V}$, well above the kink, the noise is caused by II and hole scattering in equal parts. This is due to the fact that the hole current in the source/body junction almost equals the II generated current because II generation is much larger than SRH recombination. II and hole scattering both yield shot-like noise which is strongly amplified by the floating body effect [1]. At higher frequencies this feedback effect is short-circuited by capacitive coupling and the decrease in noise with frequency is similar to the effect of a low-pass filter of the first order (i.e. lorentzian behavior).

In Fig. 6 the noise is shown for $1 \mathrm{kHz}$ versus drain bias and the total noise exhibits a peak. This peak is the result of the increase in II generation and the decrease in feedback with drain bias. The peak noise is due to II and the corresponding hole current, whereas $\mathrm{SRH}$ recombination plays a minor role. At lower drain voltages, where II plays no role and the drain current is still saturated, the noise is due to SRH recombination and relatively independent of the drain bias. Thus, even below the kink the floating body 


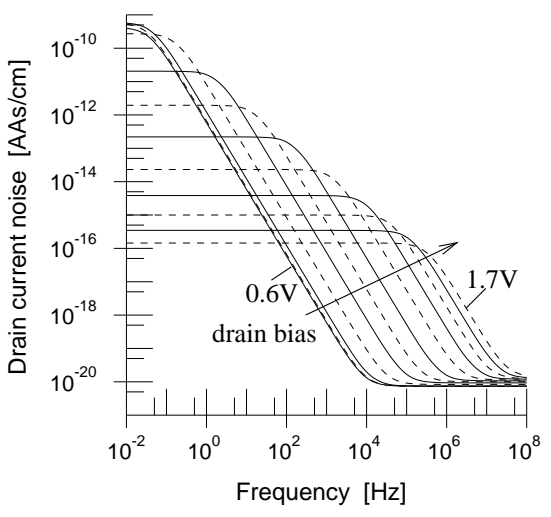

Fig. 3 Spectral intensity of the drain current fluctuations for a gate bias of $1.0 \mathrm{~V}$ and drain bias steps of $0.1 \mathrm{~V}$.

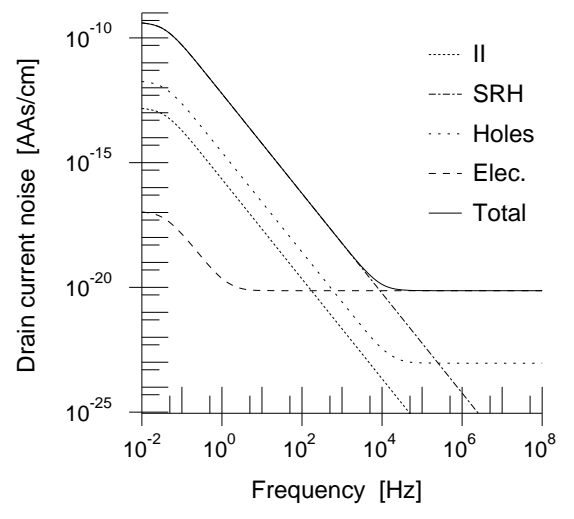

Fig. 4 Spectral intensity of the drain current fluctuations for a gate bias of $1.0 \mathrm{~V}$ and drain bias of $0.6 \mathrm{~V}$.

effect has a strong impact on noise.

\section{Conclusions}

We have presented the first 2D hydrodynamic simulations of low frequency noise in SOI devices. The physics-based simulations support the conclusions drawn in Ref. 1 based on analytical models. In addition, we have shown that noise due to traps can lead to a strong increase in noise for drain voltages below the onset of II.

\section{References}

[1] W. Jin, P. C. Chan, S. K. H. Fung, and P. K. Ko, "Shot-noise-induced excess low-frequency noise in fbating-body partially depleted SOI MOSFET's", IEEE Trans. Electron Devices, vol. 46, no. 7, pp. 1180-1185, 1999.

[2] E. Simoen, A. Mercha, J. M. Rafi, C. Claeys, N. B. Lukyanchikova, and N. Garbar, "Explaining the parameters of the electron valence-band tunneling related lorentzian noise in fully depleted soi mosfets", IEEE Electron Device Lett., vol. 24, no. 12, pp. 751-754, 2003.

[3] C. Jungemann, B. Neinhüs, and B. Meinerzhagen, "Hierarchical 2-D DD and HD noise simulations of Si and SiGe devices: Part I-Theory", IEEE Trans. Electron Devices, vol. 49, no. 7, pp. 1250-1257, 2002.

[4] C. Jungemann, B. Neinhüs, C. D. Nguyen, B. Meinerzhagen, R. W. Dutton, A. J. Scholten, and L. F. Tiemeijer, "Hydrodynamic modeling of RF noise in CMOS devices", in IEDM Tech. Dig., Washington, 2003, pp. 687-690.

[5] R. K. Mains, G. I. Haddad, and P. A. Blakey, "Simulation of GaAs IMPATT diodes including energy and velocity transport equations", IEEE Trans. Electron Devices, vol. 30, no. 10, pp. 1327-1337, 1983. 


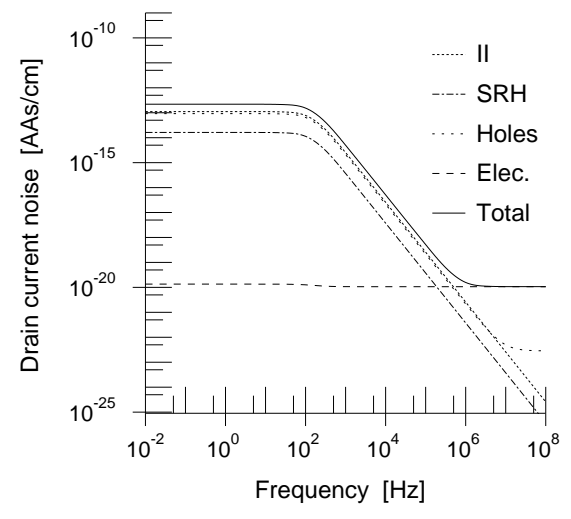

Fig. 5 Spectral intensity of the drain current fluctuations for a gate bias of $1.0 \mathrm{~V}$ and drain bias of $1.2 \mathrm{~V}$.

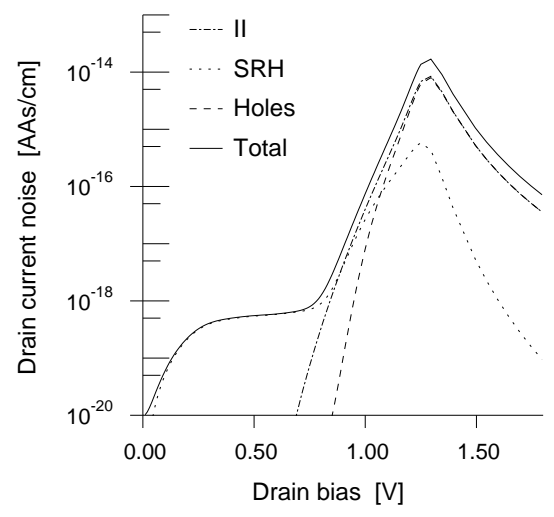

Fig. 6 Spectral intensity of the drain current fluctuations for a gate bias of $1.0 \mathrm{~V}$ and a frequency of $1 \mathrm{kHz}$.

[6] D. L. Scharfetter, "Measured dependence of lifetime upon defect density and temperature in depletion layers of epitaxial silicon diodes", in Solid-State Dev. Res. Conf., Santa Barbara, USA, 1967.

[7] F. Bonani and G. Ghione, "Generation-recombination noise modelling in semiconductor devices through population or approximate equivalent current density fluctuations", SolidState Electron., vol. 43, pp. 285-295, 1999. 